\title{
Effects of Amniotic Membrane Extract on the Hyperplastic Response of the Middle Ear Mucosa in a Bacterially-Induced Otitis Media Rat Model: A Preliminary Study
}

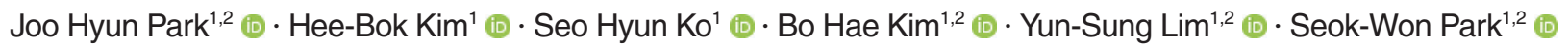 \\ Jae-Jun Song ${ }^{3}$ (i) $\cdot$ Chang Gun Cho ${ }^{1,2}$ (i) \\ ${ }^{I}$ Department of Otorhinolaryngology-Head and Neck Surgery, Dongguk University Ilsan Hospital, Dongguk University College of Medicine, \\ Goyang; ${ }^{2}$ Sensory Organ Research Institute, Dongguk University College of Medicine, Gyeongju; ${ }^{3}$ Department of Otorhinolaryngology-Head and \\ Neck Surgery, Korea University College of Medicine, Seoul, Korea
}

Objectives. Human amniotic membrane extract (AME) is known to contain numerous bioactive factors and anti-inflammatory substances. However, the anti-inflammatory effects of AME on the middle ear (ME) mucosa are unclear. This study assessed the effects of AME on the growth of the ME mucosa in response to bacterially-induced otitis media (OM).

Methods. OM was induced by inoculating nontypeable Haemophilus influenzae (NTHi) into the ME cavity of rats. ME mucosal explants were cultured in AME concentrations of $0,5,10$, or $50 \mu \mathrm{g} / \mathrm{mL}$. The area of explant outgrowth was measured in culture and analyzed at 1,3,5, and 7 days after explantation. The expression of Ki-67, mucin 5AC (MUC5AC), tumor necrosis factor- $\alpha$ (TNF- $\alpha$ ), and interleukin-10 (IL-10) in the explants was also evaluated using quantitative polymerase chain reaction (PCR) and immunocytochemistry (ICC).

Results. The NTHi-induced ME mucosa growth increased gradually over the 7-day culture period in all explants at different AME concentrations. There was a trend for mucosal growth inhibition at higher concentrations of AME, although the growth was not significantly different among the groups until day 5. The ME mucosal explants treated with the $50 \mu \mathrm{g} / \mathrm{mL}$ concentration of AME showed significantly suppressed growth on postexplantation day 7 compared with other explants on the same day. PCR and ICC staining revealed that the expression of Ki-67, MUC5AC,TNF- $\alpha$, and IL-10 further decreased in the explants with higher concentrations of AME than in those with lower concentrations of AME.

Conclusion. Our results showed that higher concentrations of AME reduced the mucosal proliferative response in bacterial $\mathrm{OM}$ in rats. These findings provide evidence that AME has an influence on the inflammatory and proliferative responses to NTHi infection in ME mucosa.

Keywords. Otitis Media; Amniotic Membrane; Haemophilus Influenzae; Rat

- Received October 28, 2019

Revised November 20, 2019

Accepted November 26, 2019

- Corresponding author: Joo Hyun Park

Department of Otorhinolaryngology-Head and Neck Surgery, Dongguk

University Ilsan Hospital, 27 Dongguk-ro, Ilsandong-gu, Goyang 10326,

Korea

Tel: +82-31-961-7436, Fax: +82-31-961-7427

E-mail: parkzzu19@dongguk.ac.kr

\section{INTRODUCTION}

Otitis media (OM) is one of the most common bacterial diseases in infancy and childhood, as $83 \%$ of children under the age of 3 years suffer from acute $\mathrm{OM}$ at least once [1,2]. Acute OM leads to more antibiotic prescriptions and surgical procedures under general anesthesia than any other condition in infancy and childhood [2]. The social burden of OM and its accompany-

Copyright ( $) 2020$ by Korean Society of Otorhinolaryngology-Head and Neck Surgery.

This is an open-access article distributed under the terms of the Creative Commons Attribution Non-Commercial License (https://creativecommons.org/licenses/by-nc/4.0)

which permits unrestricted non-commercial use, distribution, and reproduction in any medium, provided the original work is properly cited. 
ing risk of hearing loss and related complications, including linguistic ability and learning ability disorders, are well known [3-6]. Therefore, the diagnosis and proper treatment of acute $\mathrm{OM}$ are recognized as clinically and socially important problems. Although acute $\mathrm{OM}$ is a multifactorial disease to which eustachian tube dysfunction, immune function, allergy, and various environmental factors contribute, its primary cause is bacterial infection [1,7]. Eustachian tube dysfunction that is caused by an upper respiratory infection induces middle ear (ME) exudation and secondary infection by bacteria or viruses, thereby leading to acute $\mathrm{OM}$.

A major aspect of the pathology of OM is hyperplasia of the mucosa that lines the ME [8-11]. Unlike other mucosal tissues, in response to infection, the ME mucosa radically transforms from a monolayer of squamous epithelium to the full thickness of the respiratory epithelium within a few days, as has been previously shown both in animal models and in mature disease in humans [8-12]. This hyperplasia contributes significantly to the pathogenesis and symptoms of OM, through the production of mucus and other bioactive secretions, as well as by reduction of the volume of the ME cavity [13]. The proliferative response of the ME mucosa following bacterial infection in the murine $\mathrm{OM}$ has been extensively reported [13-16]. The thickness of the uninfected mucosa, which is a monolayer of simple squamous epithelium $(5-10 \mu \mathrm{m})$, increased to a peaks 48 hours after infection, with a maximum mucosal thickness of $70 \mu \mathrm{m}$ [13]. At this point, the mucosa had developed into a pseudo-columnar epithelium containing secretory and ciliated cells [13]. The ME mucosa harvested from animals infected with Haemophilus influenzae (NTHi) exhibited in vitro growth that was two to three times greater than that in mucosal samples harvested from uninfected mice [17].

The human amniotic membrane (AM) contains numerous bioactive factors and exerts anti-inflammatory and antimicrobial activities; however, these factors and their effects have not been studied in detail. The human AM exhibits anti-scarring, anti-inflammatory, immunoregulatory, and antifibrotic activities [18-

\section{H I G H L I G H T S}

- The effect of amniotic membrane extract (AME) on the growth of bacterially-induced middle ear (ME) mucosa was evaluated.

- Infected ME mucosal explants were cultured in different AME concentrations.

- The ME mucosal explants treated with $50 \mu \mathrm{g} / \mathrm{mLAME}$ showed significantly suppressed growth.

- The expression of Ki-67, mucin 5AC, tumor necrosis factor- $\alpha$, and interleukin-10 further decreased in the explants treated with higher concentrations of AME.

- Higher concentrations of AME reduced the mucosal proliferative response in bacterial otitis media in rats.
22]. Antimicrobial activity in amniotic and chorionic membranes has been previously reported [21,23-25]. Because of these properties, the AM is an excellent biomaterial for use in therapeutic applications for the skin, including burns, injuries, ophthalmic surgery, and wound healing [26-28].

In clinical settings, the application of AM has been hampered because of the need to preserve and store the entire membrane. However, the use of AM extract (AME) is a much simpler approach, which would obviate the need for surgical intervention. Many researchers have suggested that AME could provide results similar to those of AM grafting [29,30]. The literature on AME and OM is scant. In particular, the anti-inflammatory effects of AME on the ME mucosa are unclear. Recently, Yadav et al. [31] reported that AME or chorionic membrane extract (CME) significantly inhibited the growth of Streptococcus pneumoniae in planktonic form and in biofilms through its antimicrobial proteins and peptides. In this preliminary study, we aimed to evaluate the effects of AME on the hyperplastic response of bacterially-induced $\mathrm{OM}$ in infected ME mucosa using an in vitro model.

\section{MATERIALS AND METHODS}

\section{Subjects and OM induction}

To prepare the tissue culture, four Sprague-Dawley rats (7 weeks old and an average $250 \mathrm{~g}$ in weight) were used. All procedures were conducted according to the regulations of the Animal Research Institute of Medical Science, Dongguk University. The protocol of the study was approved by the Institutional Review Board (IRB No. 2016-07153).

OM was surgically induced by inoculation with a suspension of non-typeable NTHi strain 3655 into the ME cavities of the three rats through the tympanic bulla using a ventral surgical approach via an anterior neck incision [32,33]. All surgeries were performed under anesthesia, and all efforts were made to minimize the animals' suffering. The bullae of the Sprague-Dawley rats were bilaterally injected with $50 \mu \mathrm{L}$ saline containing $10^{5}$ cells/mL NTHi [32]. Following the inoculation, the tympanic membrane was visually confirmed to be intact.

\section{Preparation of AME}

Human AM matrixes were obtained from donors who had undergone caesarian section in the Department of Gynecology at Korea University Guro Hospital. The approval of the Institutional Review Board at Korea University Guro Hospital was obtained (IRB No. KUGH14239-002). Donors with gestational diabetes, preeclampsia, or infectious diseases (specifically HIV $1 \pm$ 2 , Hepatitis $B$ virus, and hepatitis $C$ virus) were excluded. AME was prepared using previously described methods $[34,35]$. AME concentrations of $0,5,10$, and $50 \mu \mathrm{g} / \mathrm{mL}$ were prepared. The extracts were stored at $-80^{\circ} \mathrm{C}$ until they were used. 


\section{ME mucosa explants culture}

At day 2 after the inoculation of the rat MEs, the animals (three experimental and a control rat) were sacrificed, and the ME mucosa was surgically harvested and divided into explants of approximately $1 \mathrm{~mm}^{2}$. To confirm the OM induction, the otoscopic exam was conducted before the animals were sacrificed. The control rat showed no signs of bacterial OM, but all rats inoculated with NTHi showed ME purulent effusion and increased vascularity. Each divided explant was individually transplanted into the wells of a 24-well culture dish containing $190 \mu \mathrm{L}$ of culture media (i.e., a mixture of Dulbecco's modified Eagle's medium and HEMs-F12 medium supplemented by $5 \%$ fetal bovine serum and $100 \mathrm{IU} / \mathrm{mL}$ penicillin, $100 \mu \mathrm{g} / \mathrm{mL}$ streptomycin, $0.4 \mu \mathrm{g} / \mathrm{mL}$ hydrocortisone, and $10^{-6} \mathrm{M}$ isoproterenol) as previously described [36]. The explants were then incubated in a humidified atmosphere of $5 \% \mathrm{CO}_{2}$ at $37^{\circ} \mathrm{C}$.

On the first day of the culture period, all wells with healthy attached OM explants were randomly divided into four groups and cultured in different concentrations of $0,5,10$, and $50 \mu \mathrm{g} / \mathrm{mL}$ of AME. In each subgroup, 20 explants were evaluated. The culture media were replaced every 3 days, and the explants were kept in culture for 7 days.

\section{Cytotoxicity measurement of AME}

After 3 days of culture, the supernatant was used to measure the lactate dehydrogenase (LDH) activity assay (LDH assay, Pierce LDH-cytotoxicity assay kit 88953 ; Thermo Scientific, Waltham, MA, USA). The cytotoxicity of the ME mucosa explants were determined by the optical absorbance read on the plate reader at $490 \mathrm{~nm}$ with background correction at $690 \mathrm{~nm}$ using a microplate spectrophotometer (Spectra max PLUS384, Sunnyvale, CA, USA). Data was converted to a percentage using a maximum LDH release control reading to create a percentage cytotoxicity and corrected for cell viability.

\section{Mucosa proliferation area evaluation}

Almost the explants were maintained in culture for 7 days. The explants that maintained a healthy appearance and remained firmly attached to the well surface throughout the entire duration of the culture were used for the data analysis. In each group, 15 explants were evaluated for mucosa proliferation area. The area of infected OM explant outgrowth was measured and analyzed on days 1, 3, 5, and 7 after explantation in culture. The explants were photographed daily using a BX61-32FA1-S09 microscope (Olympus Optical, Tokyo, Japan) to document the extent of the primary culture growth. The diameter of the explant outgrowth, which was approximately circular, was measured and its area was calculated in pixels using a microscopic computerized image analysis program (Leica Q win V3; Leica Microsystems Ltd., Bellinzona, Switzerland) and then analyzed on days 1, 3, 5, and 7 in culture (Fig. 1).

\section{Quantitative polymerase chain reaction}

The expressions of Ki-67, Mucin 5AC (MUC5AC), Tumor necrosis factor- $\alpha$ (TNF- $\alpha$ ), and interleukin-10 (IL-10) in 15 explants of each group were also evaluated using a quantitative polymerase chain reaction (PCR). Ki-67 is a nuclear protein that is associated with cellular proliferation and MUC5AC is a protein that linked to mucus hypersecretion. The total RNA was isolated and extracted from the ME mucosa of the experimental and the control rats using TRIzol Reagent (Cat. \#1596026; Invitrogen, Waltham, MA, USA) according to the manufacturer's instructions. The cDNA was synthesized using the Accupower Cycle Script RT Premix Kit (Bioneer, Seoul, Korea). A quantitative real-time PCR was performed using the AccuPower $2 \mathrm{X}$ GreenStar quantitative $\mathrm{PCR}$ (qPCR; Master Mix, Bioneer). The qPCRs of the Ki-67, MUC5AC, $T N F-\alpha$, and $I L-10$ genes were performed twice, and the glyceraldehyde 3-phosphate dehydrogenase (GAPDH) values were used to normalize the gene expression (Table 1). The gene expressions on days 1, 3, 5, and 7 after explantation were evaluated in the
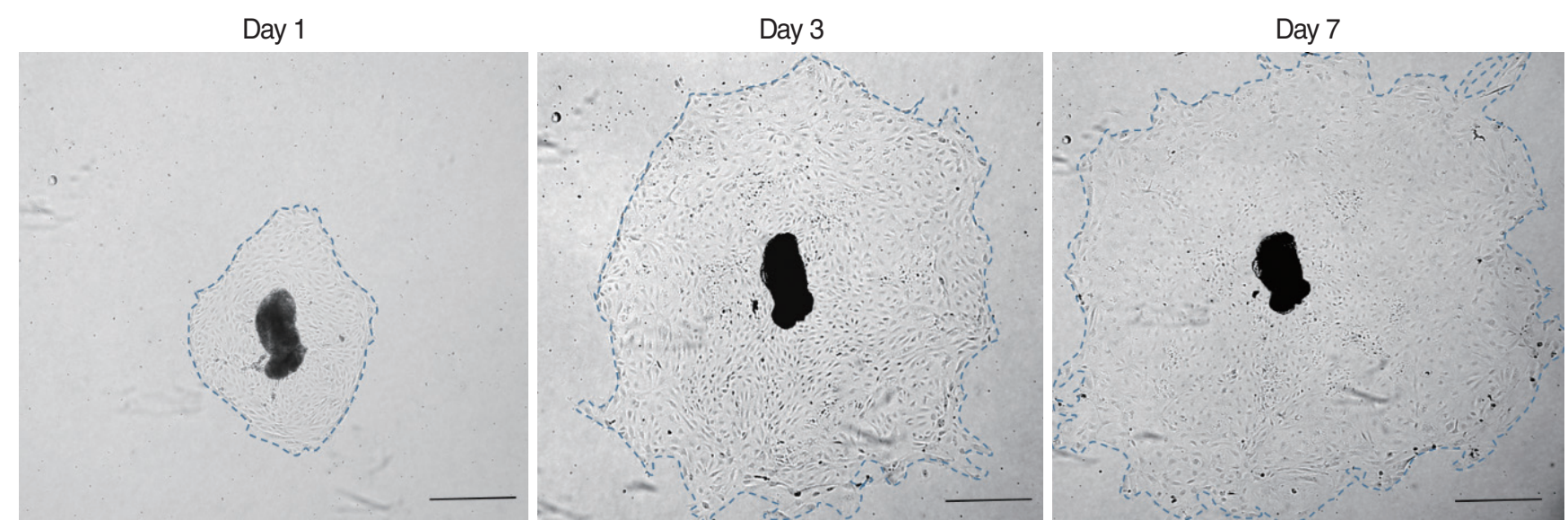

Fig. 1. Explant outgrowth evaluation. The diameter of explant outgrowth, which was approximately circular (blue dotted line), was measured, and its area was calculated in pixels using a microscopic computerized image analysis program (scale bar=100 $\mu \mathrm{m}$ ). 
Table 1. PCR primer sequences

\begin{tabular}{lll}
\hline Gene & \multicolumn{1}{c}{ Forward } & \multicolumn{1}{c}{ Reverse } \\
\hline GAPDH & 5'-TTC AAC ACC CCA GCC ATG T -3' & 5'- AGT GGT ACG ACC AGA GGC ATA CA - 3' \\
KI-67 & 5'- CCA AGA GCA TCA CAG GAA GA -3' & 5'- TTC CTC GCA TCT TTG TGA AG-3' \\
MUC5AC & 5'- GTT CTG AGA TGT CCC TCC AC -3' & 5'- GAA TGG CCA AGC TTA GGC TG -3' \\
IL-10 & 5'- GAG AGA AGC TGA AGA CCC TCT TCT -3' & 5'- TCA TTC ATG GCC TTG TAG ACA-3' \\
TNF- $-\alpha$ & 5'- ACC ACG CTC TTC TGT CTA CTG -3' & $5^{\prime}$ - CTT GGT GGT TTG CTA CGA C -3' \\
\hline
\end{tabular}

$\mathrm{PCR}$, polymerase chain reaction.

four groups that were cultured in different concentrations of AME.

\section{Immunocytochemistry}

The ME mucosa explants were cultured in culture slides (\#30104; SPL Life Sciences Co., Pocheon, Korea) for 3 days. The tissues were incubated in $100 \%$ methanol at room temperature for 5 minutes and in 4\% formaldehyde (HT501128; Sigma-Aldrich, St. Louis, MO, USA) pH 7.4 for 10 minutes at room temperature. The mucosa tissues were then heated in antigen retrieval buffer (Citrate buffer $\mathrm{Ph} 6.0$, ScyTek, CBB125) at $95^{\circ} \mathrm{C}$ for 10 minutes. The tissues were incubated with $3 \%$ bovine serum albumin (Amresco, 332-100G) in phosphate-buffered saline for 30 minutes to block the unspecific binding of the antibodies. To check the expression of Ki-67, MUC5AC, TNF- $\alpha$, and IL-10 in the ME mucosa tissue, immunocytochemical-staining antibodies for each specific antibody (TNF- $\alpha$ : ab6671, Abcam, Cambridge, UK; IL-10: MAA056Ra21, Cloud-Clone, Katy, TX, USA; MUC5AC: sc-21701, Santa Cruz Biotechnology, Dallas, TX, USA; Ki-67: ab16667, Abcam) and Hoechst stain (H6024, Sigma-Aldrich) were used. We obtained microscopic images (model no. BX53F; Olympus, Tokyo, Japan) and photographs of each slide at $400 \times$ magnification. All the detected antibodies were collected using Qwin V3 (Software Version 6; Leica Microsystems Imaging Solutions, Cambridge, UK) digital image processing and analysis software.

\section{Statistical analysis}

The statistical analysis was performed using the SPSS ver. 18.0 SPSS (SPSS Inc., Chicago, IL, USA). The criterion for statistical significance was set at $P<0.05$. All data are presented as mean \pm standard error mean. The Kruskal-Wallis $\mathrm{H}$ test was used to compare differences in the outgrowth surface areas and protein expression of the explants in the four groups on days 1,3, 5, and 7.

\section{RESULTS}

\section{Cytotoxicity measurements}

Cytotoxicity was $20 \%$ in normal culture supernatants with OM mucosa explants. AME stimulation for 3 days reduced the cell viability of ME mucosa explants, as demonstrated by increases in $\mathrm{LDH}$ activity $(5 \mu \mathrm{g} / \mathrm{mL}, 25.3 \% ; 10 \mu \mathrm{g} / \mathrm{mL}, 26.3 \% ; 50 \mu \mathrm{g} / \mathrm{mL}$,

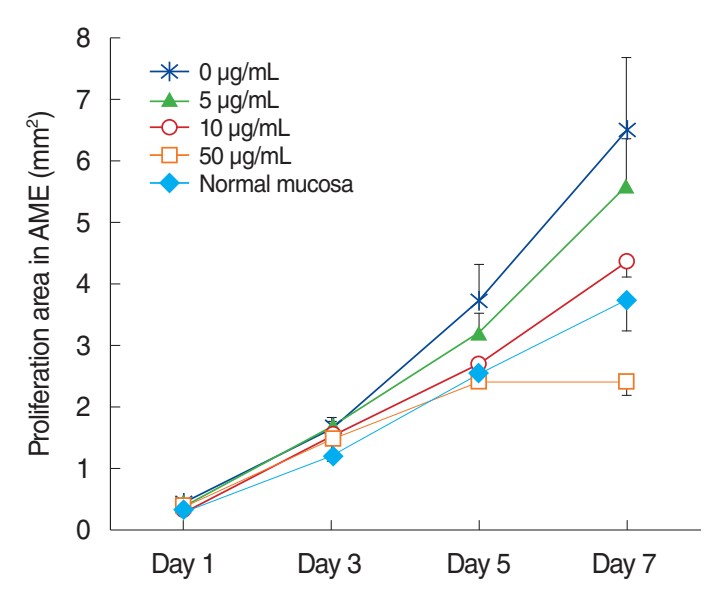

Fig. 2. Outgrowth of infected middle ear mucosal explants cultured in different concentrations of amniotic membrane extract (AME). The growth of explants with $50 \mu \mathrm{g} / \mathrm{mL}$ AME was significantly inhibited on postexplantation day 7 compared to those with lower concentrations of AME (mean \pm standard error mean).

29.1\%). A smaller increase in LDH activity was observed in stimulated explants than in non-stimulated explants. Furthermore, no statistically significant differences were observed among groups. These results suggest that AME at higher concentrations may not directly damage the OM mucosa.

\section{Proliferation area of mucosa explants}

All explants in the four groups at different AME concentrations $(0,5,10$, or $50 \mu \mathrm{g} / \mathrm{mL})$ were cultured for 7 days. In each group, 15 explants that maintained a healthy appearance and remained firmly attached to the well surface for 7 days were evaluated. All explants at different concentrations of AME grew during the culture period of 7 days. The area $\left(\mathrm{mm}^{2}\right)$ of infected OM explant outgrowth was measured and analyzed on days 1,3, 5, and 7 after explantation in culture (Fig. 2). Normal mucosa explants from non-OM rats grew less than the NTHi-infected mucosa explants in culture with $0 \mu \mathrm{g} / \mathrm{mL}$ AME on postexplantation day $1(0.27 \pm$ $0.03 \mathrm{~mm}^{2}$ vs. $\left.0.45 \pm 0.06 \mathrm{~mm}^{2}, P=0.009\right)$ and $3(1.19 \pm 0.08$ vs. $1.64 \pm 0.18, P=0.03)$. The explants in higher concentrations of AME tended to grow less than those in lower concentrations of AME. However, there was no statistically significant differences among the explants in 0,5 , and $10 \mu \mathrm{g} / \mathrm{mL}$ AME. Statistical significance was observed only on day 7 in culture with $50 \mu \mathrm{g} / \mathrm{mL}$ AME $\left(2.39 \pm 0.21 \mathrm{~mm}^{2}\right)$, which grew significantly less than the 

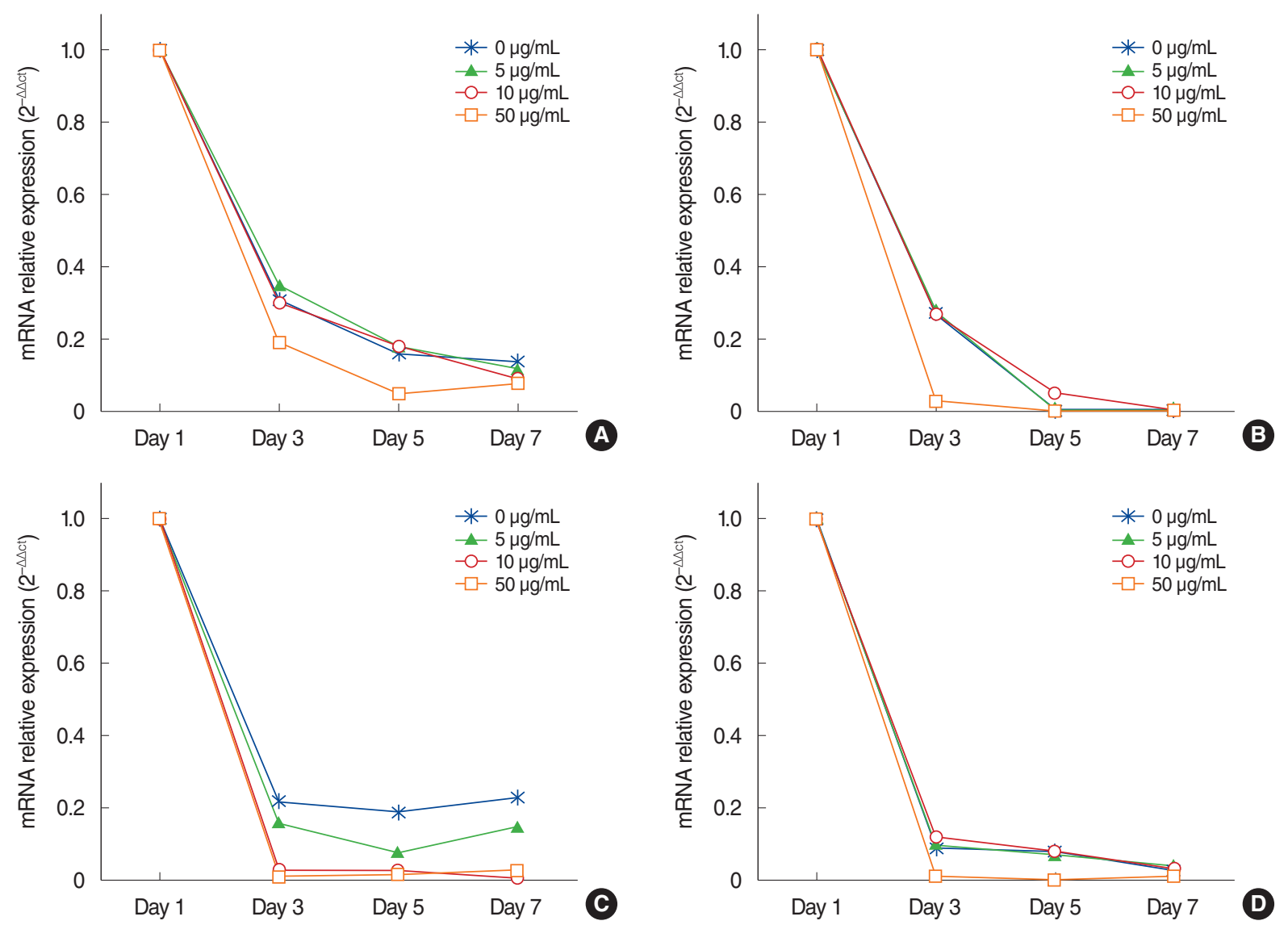

Fig. 3. Expression of Ki-67 (A), mucin 5AC (B), tumor necrosis factor- $\alpha(C)$, and interleukin-10 (D) in infected middle ear mucosa in different amniotic membrane extract concentrations at various time points after explantation.

others $\left(0 \mu \mathrm{g} / \mathrm{mL}, 6.49 \pm 1.18 \mathrm{~mm}^{2} ; 5 \mu \mathrm{g} / \mathrm{mL}, 5.58 \pm 0.79 \mathrm{~mm}^{2}\right.$; $\left.10 \mu \mathrm{g} / \mathrm{mL}, 4.36 \pm 0.26 \mathrm{~mm}^{2}\right)$ on postexplantation day $7(P<0.001)$.

\section{Expression of Ki-67, MUC5AC, TNF- $\alpha$, and IL-10 in infected ME mucosa}

The mRNA expression of Ki-67 decreased rapidly until 3 days after explantation in all groups, and groups with higher concentrations of AME tended to show greater decreases (Fig. 3). Statistical significance was observed on days $3(P=0.038)$ and $5(P=$ 0.038 ) in culture with $50 \mu \mathrm{g} / \mathrm{mL}$ AME. The immunocytochemistry (ICC) results showed similar patterns to the qPCR results (Fig. 4).

The mRNA expression of MUC5AC showed a similar pattern to that of Ki-67 (Fig. 3). Almost no expression of MUC5AC was observed in the mucosa with $50 \mu \mathrm{g} / \mathrm{mLAME}$ after postexplantation day 3 (Fig. 3). However, no statistically significant differences were found among groups with different AME concentrations. ForTNF- $\alpha$ and IL-10 expression, the mRNA expression and ICC results also showed a rapid decrease after explantation, and explants with higher concentration of AME tended to show greater decreases (Figs. 3 and 4). Explants with $50 \mu \mathrm{g} / \mathrm{mL}$ AME exhibited nearly no IL-10 expression after the 3 rd day $(P=0.052)$. The changes in IL-10 expression on day $7(P=0.039)$ and TNF- $\alpha$ on day 5 and 7 were statistically significant $(P=0.038)$.

\section{DISCUSSION}

In humans, the AM is the inner fetal membrane, which is located in the inner side of the placenta and is known to exert antiapoptotic, antiangiogenic, antimicrobial, and anti-inflammatory effects on epithelial cells [18-22,37]. Because of these properties, the AM is a potential therapeutic biomaterial that could encourage wound healing and anti-inflammatory responses in various clinical areas, especially ophthalmologic and dermatologic diseases and injuries [26-28]. AME is a simple way to apply the AM as a biomaterial in clinical settings. Several researchers have suggested that AME and AM grafting are comparable in terms of their anti-inflammatory effects $[29,30,37]$. Human AME also has been shown to contain human neutrophil peptides, lysozyme, calprotectin, ubiquitin, and several bactericidal proteins that show antiapoptotic and anti-inflammatory effects [31,37]. Moreover, it was reported that AME induced morphological changes in macrophages, enhanced cell apoptosis, and downregulated the 


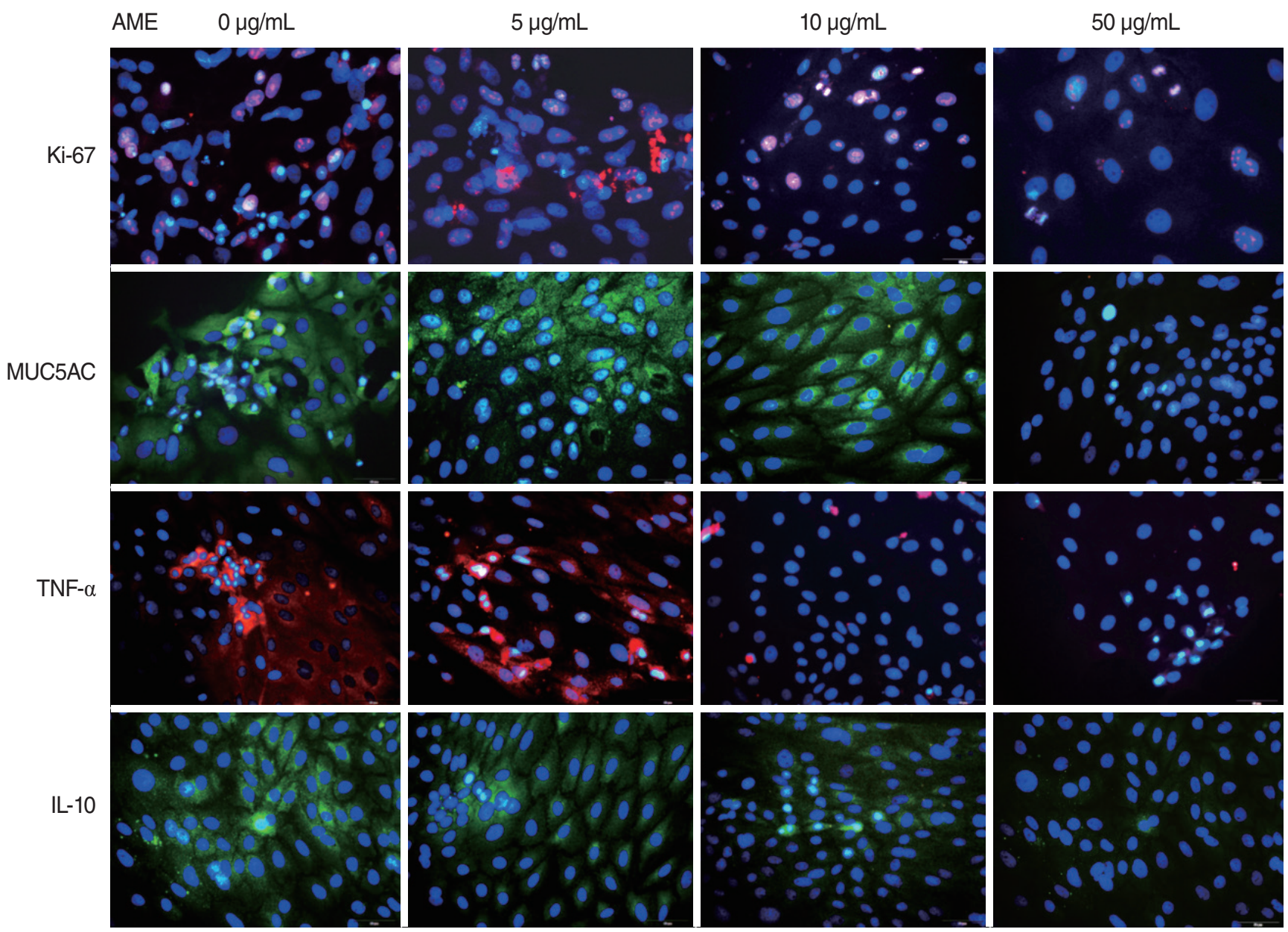

Fig. 4. Immunocytochemical staining of Ki-67, mucin 5AC (MUC5AC), tumor necrosis factor- $\alpha$ (TNF- $\alpha$ ), and interleukin-10 (IL-10) in infected middle ear mucosa explants on postexplantation day 3. Immunocytochemistry shows decreased expression in the explants with higher concentrations of amniotic membrane extract (AME; $\times 400$, scale bar $=50 \mu \mathrm{m})$.

antiapoptotic nuclear factor kappa-light-chain-enhancer of activated $B$ cells $(\mathrm{NF}-\mathrm{\kappa} \mathrm{B})$ signaling pathway in vitro [38]. However, the precise mechanisms through which the AM and AME exert effects on several cell types and the role of soluble factors in the function of AM and AME are still poorly understood. We hypothesized that AME plays an anti-inflammatory role in ME epithelial cells in ME infections.

Few previous studies have explored the effects of AME on OM. Yadav et al. [31] reported that AME and CME significantly inhibited the growth of $S$. pneumoniae in planktonic form and in biofilms through its antimicrobial proteins and peptides. Treatment with AME and CME showed significant inhibition of S. pneumoniae growth. Regarding bactericidal effects, the combined application of AME/CME and antibiotic solution showed a synergistic effect on the inhibition of bacterial infection in the ME.

In this study, we evaluated the effects of AME, focusing on the hyperplastic response of ME mucosa, which is the major pathology in OM [8-11], in response to bacterially-induced OM in an in vitro model. The AME components seemed to suppress bacte- rially-stimulated ME mucosal growth in a dose-dependent manner, although statistical significance was observed only on day 7 in a culture with $50 \mu \mathrm{g} / \mathrm{mLAME}$. The ME mucosa has been shown to radically transform from a monolayer of squamous epithelium to a full-thickness pseudo-columnar epithelium containing secretory and ciliated cells [13]. These pathological changes induce mucus production and bioactive secretions in response to infections [8-13]. Ki-67 and MUC5AC were evaluated based on the pathology of OM. Ki-67 is a nuclear protein that is associated with cellular proliferation and used as a cellular marker of cell proliferation [39]. MUC5AC has been linked to mucus hypersecretion [40]. The expression of Ki-67, MUC5AC, TNF- $\alpha$, and IL10 decreased rapidly until day 3 after explantation. This finding is most likely linked to the fact that the present research was an in vitro study. Expression in cultures with higher concentrations of AME showed steeper declines. These results showed that AME had a dose-dependent inhibitory effect on the growth of infected ME mucosa.

It is important to note that caution should be used in inter- 
preting these results. The results of this study did not definitively show the effects of AME on the hyperplastic response of bacterially-stimulated $\mathrm{ME}$ growth because of several limitations. The in vitro culture model of mucosal growth in infected ME may not be identical to in vivo responses. Moreover, the most suitable AME concentration was not determined. AME concentrations between 10 and $50 \mu \mathrm{g} / \mathrm{mL}$ were not analyzed in this study, although they may have shown different patterns. Hence, studies should explore the possibility that more precisely targeted AME concentrations between 10 and $50 \mu \mathrm{g} / \mathrm{mL}$ could suppress mucosal growth. Further research through in vivo animal studies should be conducted to clarify the function of AME in the occurrence and progression of OM. Further experiments are needed to confirm the components of AME that show anti-inflammatory and antiproliferative effects. In addition, only AME was evaluated in this study. A previous study showed that CME greatly enhanced osteogenesis and indicated that AME and CME exerted differential effects on osteogenesis because of their different compositions of growth factors involved in regeneration [35]. Hence, the effects of CME on the ME mucosa in response to infection would be an interesting topic for further studies.

Despite these limitations, our results are meaningful because studies on AME and OM have been rare. Moreover, our results provide data supporting the possibility that AME may exert antiproliferative and anti-inflammatory effects on infected ME mucosa. This study showed that AME reduced the mucosal proliferative response in bacterially-induced OM in rats in a dose-dependent manner. These findings provide evidence that AME may have an influence on the inflammatory and proliferative responses to NTHi infection in ME mucosa. Our study results may help to provide a basis for future studies of the effect of AME on OM.

\section{CONFLICT OF INTEREST}

No potential conflict of interest relevant to this article was reported.

\section{ACKNOWLEDGMENTS}

This work was supported by the Dongguk University Research Fund of 2016 (K-2016-G00002-00011).

\section{ORCID}

Joo Hyun Park

Hee-Bok Kim

Seo Hyun Ko

Bo Hae Kim https://orcid.org/0000-0002-1499-3328

https://orcid.org/0000-0001-9084-9734

https://orcid.org/0000-0003-3558-6163

https://orcid.org/0000-0002-4645-0678
Yun-Sung Lim

Seok-Won Park

Jae-Jun Song

Chang Gun Cho

https://orcid.org/0000-0002-5997-6193

https://orcid.org/0000-0002-8488-9091

https://orcid.org/0000-0002-7103-8896 https://orcid.org/0000-0003-4262-2150

\section{AUTHOR CONTRIBUTIONS}

Conceptualization: JHP, CGC. Data curation: HBK, SHK, JJS. Formal analysis: JHP, HBK, SHK. Funding acquisition: JHP. Methodology: JHP, HBK, CGC. Project administration: JHP, HBK, SHK, JJS. Visualization: JHP, HBK. Writing-original draft: JHP.Writing-review \& editing: JHP, HBK, BHK, YSL, SWP, CGC.

\section{REFERENCES}

1. Coticchia JM, Chen M, Sachdeva L, Mutchnick S. New paradigms in the pathogenesis of otitis media in children. Front Pediatr. 2013 Dec; 1:52.

2. Rovers MM, Schilder AG, Zielhuis GA, Rosenfeld RM. Otitis media. Lancet. 2004 Feb;363(9407):465-73.

3. Infante-Rivard C, Fernandez A. Otitis media in children: frequency, risk factors, and research avenues. Epidemiol Rev. 1993;15(2):44465.

4. Klein JO.The burden of otitis media. Vaccine. 2000 Dec;19 Suppl 1: S2-8.

5. Al Khabori M, Khandekar R. The prevalence and causes of hearing impairment in Oman: a community-based cross-sectional study. Int J Audiol. 2004 Sep;43(8):486-92.

6. Heikkinen T, Chonmaitree T. Importance of respiratory viruses in acute otitis media. Clin Microbiol Rev. 2003 Apr;16(2):230-41.

7. Teele DW, Klein JO, Chase C, Menyuk P, Rosner BA. Otitis media in infancy and intellectual ability, school achievement, speech, and language at age 7 years. Greater Boston Otitis Media Study Group. J Infect Dis. 1990 Sep;162(3):685-94.

8. Lim DJ, Birck H. Ultrastructural pathology of the middle ear mucosa in serous otitis media. Ann Otol Rhinol Laryngol. 1971 Dec;80(6): 838-53.

9. Tos M, Caye-Thomasen P. Mucous glands in the middle ear: what is known and what is not. ORL J Otorhinolaryngol Relat Spec. 2002 Mar-Apr;64(2):86-94.

10. Bluestone CD, Klein JO. Otitis media with effusion, atelectasis, and Eustachian tube dysfunction. In: Bluestone CD, Stool SE, Arjona S, editors. Pediatric otolaryngology. Philadelphia (PA): Saunders; 1983. p. 356-512.

11. Schuknecht HF. Pathology of the ear. Philadelphia (PA): Lea \& Febiger; 1993.

12. Ryan AF, Catanzaro A, Wasserman SI, Harris JP. Secondary immune response in the middle ear: immunological, morphological, and physiological observations. Ann Otol Rhinol Laryngol. 1986 May-Jun;95 (3 Pt 1):242-9.

13. Cho CG, Pak K, Webster N, Kurabi A, Ryan AF. Both canonical and non-canonical NF- $\mathrm{KB}$ activation contribute to the proliferative response of the middle ear mucosa during bacterial infection. Innate Immun. 2016 Nov;22(8):626-34.

14. Hernandez M, Leichtle A, Pak K, Ebmeyer J, Euteneuer S, Obonyo $\mathrm{M}$, et al. Myeloid differentiation primary response gene 88 is required for the resolution of otitis media. J Infect Dis. 2008 Dec;198(12): 1862-9. 
15. Leichtle A, Hernandez M, Pak K, Yamasaki K, Cheng CF, Webster $\mathrm{NJ}$, et al. TLR4-mediated induction of TLR2 signaling is critical in the pathogenesis and resolution of otitis media. Innate Immun. 2009 Aug;15(4):205-15.

16. Ryan AF, Ebmeyer J, Furukawa M, Pak K, Melhus A, Wasserman SI, et al. Mouse models of induced otitis media. Brain Res. 2006 May; 1091(1):3-8.

17. Palacios SD, Pak K, Kayali AG, Rivkin AZ,Aletsee C, Melhus A, et al. Participation of Ras and extracellular regulated kinase in the hyperplastic response of middle-ear mucosa during bacterial otitis media. J Infect Dis. 2002 Dec;186(12):1761-9.

18. Silini A, Parolini O, Huppertz B, Lang I. Soluble factors of amnionderived cells in treatment of inflammatory and fibrotic pathologies. Curr Stem Cell Res Ther. 2013 Jan;8(1):6-14.

19. Ricci E, Vanosi G, Lindenmair A, Hennerbichler S, Peterbauer-Scherb A, Wolbank S, et al. Anti-fibrotic effects of fresh and cryopreserved human amniotic membrane in a rat liver fibrosis model. Cell Tissue Bank. 2013 Sep;14(3):475-88.

20. Hao Y, Ma DH, Hwang DG, Kim WS, Zhang F. Identification of antiangiogenic and antiinflammatory proteins in human amniotic membrane. Cornea. 2000 May;19(3):348-52.

21. Faulk WP, Matthews R, Stevens PJ, Bennett JP, Burgos H, Hsi BL. Human amnion as an adjunct in wound healing. Lancet. 1980 May; 1(8179):1156-8.

22. Bailo M, Soncini M,Vertua E, Signoroni PB, Sanzone S, Lombardi G, et al. Engraftment potential of human amnion and chorion cells derived from term placenta. Transplantation. 2004 Nov;78(10):1439-48.

23. Talmi YP, Sigler L, Inge E, Finkelstein Y, Zohar Y. Antibacterial properties of human amniotic membranes. Placenta. 1991 MayJun;12(3):285-8.

24. Kjaergaard N, Hein M, Hyttel L, Helmig RB, Schonheyder HC, Uldbjerg N, et al. Antibacterial properties of human amnion and chorion in vitro. Eur J Obstet Gynecol Reprod Biol. 2001 Feb;94(2): 224-9.

25. Sangwan VS, Basu S.Antimicrobial properties of amniotic membrane. Br J Ophthalmol. 2011 Jan;95(1):1-2.

26. Gheorghe A, Pop M, Burcea M, Serban M. New clinical application of amniotic membrane transplant for ocular surface disease. J Med Life. 2016 Apr-Jun;9(2):177-9.

27. Mohan R, Bajaj A, Gundappa M. Human amnion membrane: potential applications in oral and periodontal field. J Int Soc Prev Community Dent. 2017 Jan-Feb;7(1):15-21.

28. Mamede AC, Carvalho MJ,Abrantes AM, Laranjo M, Maia CJ, Botel- ho MF. Amniotic membrane: from structure and functions to clinical applications. Cell Tissue Res. 2012 Aug;349(2):447-58.

29. Guo Q, Hao J, Yang Q, Guan L, Ouyang S, Wang J. A comparison of the effectiveness between amniotic membrane homogenate and transplanted amniotic membrane in healing corneal damage in a rabbit model. Acta Ophthalmol. 2011 Jun;89(4):e315-9.

30. Mahbod M, Shahhoseini S, Khabazkhoob M, Beheshtnejad AH, Bakhshandeh H, Atyabi F, et al. Amniotic membrane extract preparation: what is the best method? J Ophthalmic Vis Res. 2014 Jul-Sep; 9(3):314-9.

31. Yadav MK, Go YY, Kim SH, Chae SW, Song JJ. Antimicrobial and antibiofilm effects of human amniotic/chorionic membrane extract on Streptococcus pneumoniae. Front Microbiol. 2017 Oct;8:1948.

32. Cho CG, Gong SH, Kim HB, Song JJ, Park JH, Lim YS, et al. Role of group 3 innate lymphoid cells during experimental otitis media in a rat model. Int J Pediatr Otorhinolaryngol. 2016 Sep;88:146-52.

33. Yu GH, Kim HB, Ko SH, Kim YW, Lim YS, Park SW, et al. Expression of surfactant Protein-A in the Haemophilus influenzae-induced otitis media in a rat model. Int J Pediatr Otorhinolaryngol. 2018 Sep; 112:61-6.

34. GoYY, Kim SE, Cho GJ, Chae SW, Song JJ. Promotion of osteogenic differentiation by amnion/chorion membrane extracts. J Appl Biomater Funct Mater. 2016 May;14(2):e171-80.

35. Go YY, Kim SE, Cho GJ, Chae SW, Song JJ. Differential effects of amnion and chorion membrane extracts on osteoblast-like cells due to the different growth factor composition of the extracts. PLoS One. 2017 Aug;12(8):e0182716.

36. Kim HA, Kim S, Chang SH, Hwang HJ, Choi YN. Anti-arthritic effect of ginsenoside $\mathrm{Rb} 1$ on collagen induced arthritis in mice. Int Immunopharmacol. 2007 Oct;7(10):1286-91.

37. He H, LiW, Chen SY, Zhang S, Chen YT, Hayashida Y, et al. Suppression of activation and induction of apoptosis in RAW264.7 cells by amniotic membrane extract. Invest Ophthalmol Vis Sci. 2008 Oct; 49(10):4468-75.

38. LiW, He H, Kawakita T, Espana EM,Tseng SC. Amniotic membrane induces apoptosis of interferon-gamma activated macrophages in vitro. Exp Eye Res. 2006 Feb;82(2):282-92.

39. Scholzen T, Gerdes J. The Ki-67 protein: from the known and the unknown. J Cell Physiol. 2000 Mar;182(3):311-22.

40. Wang G, Xu Z, Wang R, Al-Hijji M, Salit J, Strulovici-Barel Y, et al. Genes associated with MUC5AC expression in small airway epithelium of human smokers and non-smokers. BMC Med Genomics. 2012 Jun;5:21. 